\title{
Extensão de Cadeia em Poli(Naftalato de Etileno) pelo Fosfito de Trifenila
}

\author{
Marcos L. Dias, Alexandre P. F. Silva \\ Instituto de Macromoléculas Professora Eloisa Mano, UFRJ
}

Resumo: O processamento reativo de poli(naftalato de etileno) (PEN) na presença de fostito de trifenila (TPP) em um misturador interno acoplado ao reômetro de torque foi investigado. $\mathrm{O}$ acompanhamento do torque e da temperatura durante o processamento revelou a ocorrência de reações de extensão de cadeia. A massa molecular do poliéster aumentou com o tempo de processamento, passando por um máximo, após o qual começa a decrescer como resultado de degradação molecular acelerada pela presença de subprodutos da extensão de cadeia. A intensidade das reações de extensão de cadeia aumenta com o aumento do teor de TPP, da temperatura e do cisalhamento. Tanto a extensão de cadeia quanto a degradação ocorrem mais lentamente para o PEN que para o poli(tereftalato de etileno). A presença de TPP no material provoca modificações na $\mathrm{T}_{\mathrm{g}}$, devido a um efeito de plastificação, e aumenta a velocidade de cristalização.

Palavras-chave: Extensão de cadeia, fosfitos, poli(naftalato de etileno), PEN, PET.

\section{Chain Extention of Poly(ethylene naphthalate) with Triphenylphosphite}

Abstract: The reactive processing of poly(ethylene naphthalate) (PEN) in presence of triphenylphosphite (TPP) was investigated. An internal mixer coupled to a torque rheometer was used to monitor changes of molecular mass and temperature during processing. The torque curves revealed that chain extension reactions took place. During the processing, there is an increase in the molecular weight of the polyester, which reaches a maximum and then decreases due to molecular degradation, accelerated by the presence of chain extention by-products. The intensity of the chain extention reaction increases when TPP content, temperature and shear rate rise. Chain extension and degradation rate are slower in PEN than in poly(ethylene terephthalate). The presence of low molecular weight by-products of chain extension in PEN causes changes in the $\mathrm{T}_{\mathrm{g}}$, due to plasticization effects, and increase the crystallization rate.

Keywords: Chain extention, polyesters, phosphites, PEN, PET.

\section{Introdução}

O poli(naftalato de etileno) (PEN) é um poliéster obtido pela policondensação de etileno glicol e ácido 2,6-naftaleno-dicarboxílico em processo similar ao empregado na produção de poli(tereftalato de etileno) (PET). O polímero apresenta como propriedades principais transparência, baixa permeabilidade à gases e grande resistência mecânica, especialmente quando estirado, o que faz com que possa ser utilizado em substituição ao PET em uma série de aplicações ${ }^{[1,2]}$. Por exemplo, o PEN pode ser empregado em embalagens transparentes para produtos sen- síveis à oxidação, devido à melhor impermeabilidade ao oxigênio molecular que o PET; é também adequado quando o produto é envasado à quente ou é submetido à esterilização por pasteurização, devido à melhor estabilidade dimensional em temperaturas mais elevadas.

Em todos esses usos, PEN com maior massa molecular é necessário. O PEN com essa característica tem sido obtido comercialmente pela póscondensação no estado sólido ${ }^{[3,4]}$. Esse processo tem inúmeras vantagens sobre o processo de polimerização no estado fundido que operam em altas temperaturas, que pode causar a decomposição do

Autor para correspondência: Marcos L. Dias, Instituto de Macromoléculas Prof. Eloisa Mano/UFRJ, C.P.: 68252, CEP: 21945-970, Rio de Janeiro, RJ. E-mail:mldias@ima.ufrj.br 
poliéster e geração de subprodutos indesejáveis. Contudo, para que altos pesos moleculares sejam alcançados na polimerização no estado sólido, longos tempos de reação são necessários, já que o processo é lento. Além disso, o processo normalmente requer que o polímero com baixo peso molecular, na forma de partículas ou grão, passe por longo processo de cristalização antes da polimerização no estado sóli$\mathrm{do}^{[5]}$. Assim, métodos mais eficientes e rápidos para aumentar o peso molecular são desejáveis.

$\mathrm{O}$ aumento do peso molecular de poliésteres e poliamidas pode ser obtido de forma rápida pelo uso de extensores de cadeia ${ }^{[6-14]}$. Os extensores de cadeia são normalmente moléculas de baixa massa molar capazes de reagir com os grupos terminais de cadeia desses polímeros de condensação, promovendo a ligação entre cadeias. O processo ocorre normalmente em temperaturas, onde o material encontra-se no estado fundido, podendo ser conduzido em extrusora. Dependendo do tipo de reação, o processo de extensão de cadeia pode ser muito rápido, resultando em aumento instantâneo da viscosidade do fundido.

Vários tipos de compostos químicos podem ser empregados como agentes extensores de cadeia para poliésteres e poliamidas. Alguns desses compostos são as oxazolinas, os dianidridos, os diepóxidos, e alguns compostos organofosforados ${ }^{[6]}$.

O processo de extensão de cadeia de poliésteres e poliamidas tem sido investigado por nosso grupo de pesquisa ${ }^{[13-16]}$. Neste trabalho são apresentados os resultados do estudo sobre a extensão de cadeia de PEN, utilizando-se fostito de trifenila (TPP) como agente extensor. $\mathrm{O}$ estudo foi conduzido em câmara de mistura de um reômetro de torque que simula as condições de um reator químico capaz de promover a mistura do polímero com o extensor de cadeia no estado fundido, com monitoramento do torque e da temperatura com o tempo.

\section{Experimental}

\section{Materiais}

PEN produzido em escala piloto pela Rhodia-Ster foi fornecido na forma de grânulos amorfos $\left(\overline{\mathrm{M}}_{\mathrm{w}}=37900, \mathrm{~T}_{\mathrm{m}}=254^{\circ} \mathrm{C}\right)$. Poli(tereftalato de etileno) grau garrafa foi fornecido pela Hoescht $\left(\overline{\mathrm{M}}_{\mathrm{w}}=39000\right.$, $\mathrm{T}_{\mathrm{m}}=247^{\circ} \mathrm{C}$ ). $\mathrm{O}$ fosfito de trifenila (Aldrich) e acetona P.A. (Vetec) foram usados como recebidos.

\section{Processamento}

As amostras foram processadas em um reômetro de torque Haake Reocord 9000 equipado com uma câmara de mistura Reomix 600. Cerca de $50 \mathrm{~g}$ de amostra, previamente seca a $160^{\circ} \mathrm{C}$ por $16 \mathrm{~h}$, foram introduzidas na câmara de mistura, já regulada para a temperatura desejada $\left(275^{\circ} \mathrm{C}\right)$, e processada a $60 \mathrm{rpm}$. Essas condições foram empregadas na maioria dos casos, exceto nos experimentos realizados para verificação da influência da temperatura e da velocidade de rotação dos rotores do misturador, onde a temperatura e a velocidade foram variadas. A adição do extensor de cadeia por meio de seringa ocorreu após 5 minutos de processamento, quando o material estava completamente fundido e o torque estável. O torque e a temperatura foram registrados durante o processamento.

\section{DSC}

As análises de calorimetria diferencial de varredura foram realizadas em um equipamento Perkin Elmer DSC-7 na faixa de 50 a $290^{\circ} \mathrm{C}$. As amostras foram inicialmente aquecidas a $20^{\circ} \mathrm{C} / \mathrm{min}$, resfriadas a aproximadamente $200^{\circ} \mathrm{C} / \mathrm{min}$, reaquecidas a $10^{\circ} \mathrm{C} / \mathrm{min}$ e finalmente resfriadas a $10^{\circ} \mathrm{C} / \mathrm{min}$. Por meio do tratamento térmico mencionado, foi possível determinar a temperatura de transição vítrea $\left(\mathrm{T}_{\mathrm{g}}\right)$, a temperatura de fusão cristalina $\left(\mathrm{T}_{\mathrm{m}}\right)$ e as temperaturas de cristalização no aquecimento $\left(\mathrm{T}_{\text {ch }}\right)$ e no resfriamento $\left(\mathrm{T}_{\mathrm{cc}}\right)$.

\section{SEC}

O peso molecular e a polidispersão das amostras de PEN foram determinadas por cromatografia de exclusão por tamanho (SEC), empregando-se um conjunto de 3 colunas Ultrastyragel (2 lineares e uma $500 \AA$ ), a mistura de clorofórmio/1,1,1,3,3,3hexafluoroisopropanol $=98 / 2(\mathrm{p} / \mathrm{v})$ como eluente e padrões de poliestireno monodispersos para calibração relativa. Utilizou-se concentração de polímero de $0,2 \%(\mathrm{p} / \mathrm{v})$, vazão de $1 \mathrm{~mL} / \mathrm{min}$ e volume de injeção de $200 \mu \mathrm{L}$.

\section{Extração}

Após o processamento, todas as amostras foram trituradas em moinho de facas IKA Mikro-FeinmuhleCulatti, resultando em material particulado com cerca de $2 \mathrm{~mm}$ de diâmetro. Parte de cada amostra pulverizada foi submetida à extração com acetona por $48 \mathrm{~h}$ em 
Soxhlet, para remoção dos subprodutos de baixa massa molar provenientes da reação de extensão de cadeia.

\section{Resultados e Discussão}

\section{Processamento de PEN em presença de TPP}

Quando o PEN é processado em misturador HAAKE a $275^{\circ} \mathrm{C}$ durante $30^{\prime}$ sem nenhum aditivo, observa-se uma queda nos valores de torque com o tempo. A curva torque versus tempo (Figura 1) mostra que o torque do material fundido diminui "suavemente”. Esta diminuição está relacionada com a degradação mecânica e térmica causada pelo cisalhamento e pela elevada temperatura do experimento.

Com a adição de $2 \%$ em massa de TPP, é observado um aumento significativo dos valores de torque, já a partir de 2'após a sua adição. Na Figura 1, o tempo zero corresponde à injeção do aditivo na câmara de mistura, o que ocorreu após completa fusão do polímero (5'). Este procedimento foi adotado em todo trabalho.

O comportamento do torque foi atribuído a reações de extensão de cadeia que ocorrem entre os grupos terminais de poliésteres e fosfitos ${ }^{[8,13-15]}$, aumentando o tamanho das cadeias macromoleculares. $\mathrm{O}$ aumento do tamanho das cadeias, que eleva a viscosidade do meio e, consequentemente o torque, supera os efeitos causados pela degradação observada no processamento do polímero puro.

É interessante ressaltar o aparecimento de um ponto de máximo em torno de 6' de processamento. À partir deste torque máximo, os valores vão diminuindo continuamente com o tempo, devido a velocidade de cisão de cadeias tornar-se mais rápida que a velocidade de extensão de cadeia. A queda tão acentuada destes valores ocorre pela ação dos subprodutos da reação de extensão de cadeia, principalmente resíduos fenólicos ${ }^{[17]}$,

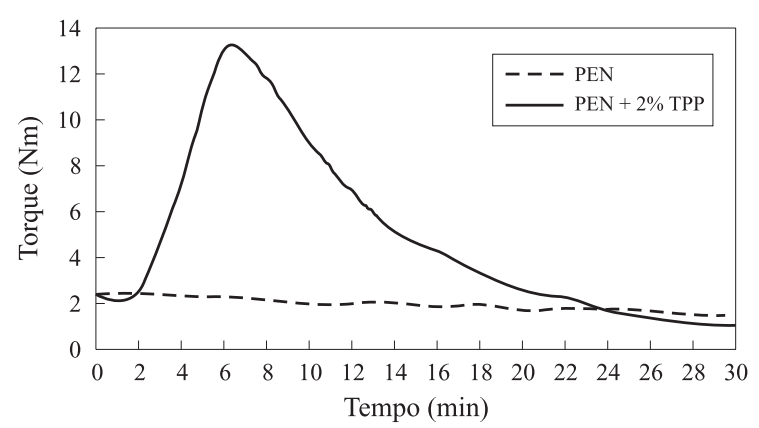

Figura 1. Curva de torque versus tempo de processamento do PEN a $275^{\circ} \mathrm{C}$ e $60 \mathrm{rpm}$.
1. Extensão de cadeia
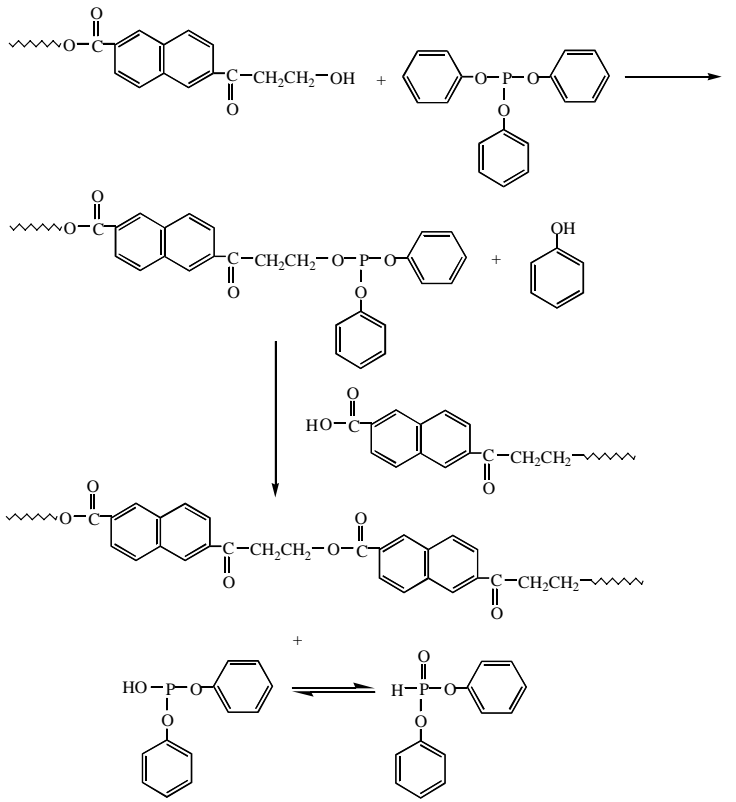

2. Degradação do PEN por alcoólise

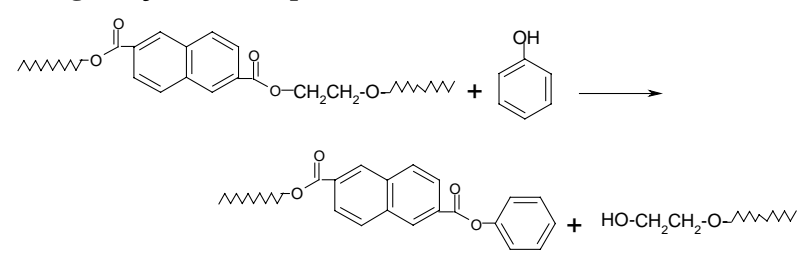

Esquema 1. Reações de extensão de cadeia de PEN com TPP e alcoólise por resíduos gerados durante o processamento sob altas temperaturas

que podem promover reações de alcoólise (Esquema 1), que rompem as ligações éster da cadeia, fazendo com que o torque seja reduzido rapidamente. A reação que provavelmente ocorre no processo de extensão de cadeia ${ }^{[13]}$ também é mostrado no Esquema 1. Após 24', os valores de torque da curva de processamento de PEN na presença de TPP são menores do que os valores obtidos no mesmo tempo, quando o polímero é processado em condições idênticas, sem o aditivo.

\section{Peso molecular durante o processamento}

Com o objetivo de verificar se os aumentos de torque observados correspondiam a aumentos no peso molecular, o PEN foi processado na presença de $2 \%$ de TPP, em câmara de mistura, empregando-se diversos tempos de processamento. Cada amostra obtida foi posteriormente analisada por SEC.

A curva de peso molecular versus tempo apresentou perfil semelhante ao verificado para a curva de torque versus tempo (Figura 2). Com a introdução de TPP, após 5' de processamento, ocorreu um aumento 


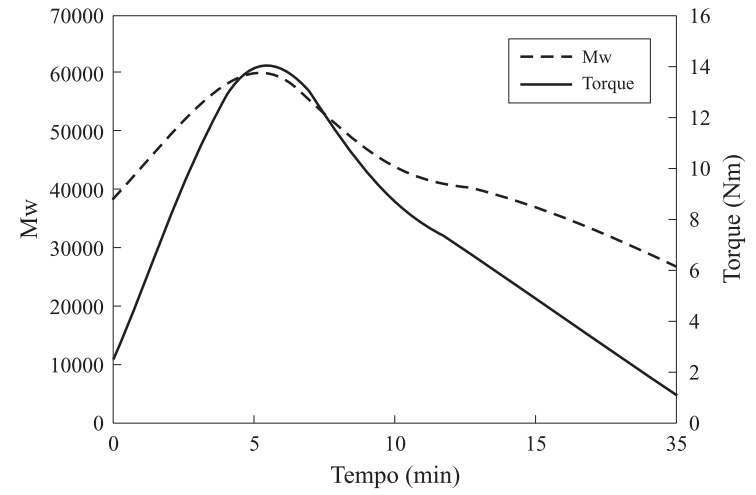

Figura 2. Torque e peso molecular versus tempo de processamento, a $275^{\circ} \mathrm{C}$ e $60 \mathrm{rpm}$, de PEN aditivado com $2 \%$ de TPP.

expressivo do peso molecular até atingir um ponto de máximo, que coincide com o ponto de máximo observado para o torque. A partir deste máximo, os valores de peso molecular são reduzidos à medida que o tempo de processamento é aumentado, até alcançar valores menores que os determinados para o polímero puro.

Este resultado confirma a ocorrência das reações de extensão de cadeia pela adição de TPP ao poliéster, e sugere uma correlação direta de aumento de torque com o aumento de peso molecular. A Tabela 1 mostra os resultados de pesos moleculares e polidispersão obtidos através de análise por SEC.

A análise dos resultados de polidispersão durante o processo revelou interessante comportamento. $\mathrm{Na}$ amostra processada sem TPP por 5', o valor de $\bar{M} w$ diminue em virtude, principalmente, da quebra de ligações das moléculas de pesos moleculares mais elevados. Essa fração de moléculas que se tornaram menores, enriquece as frações de pesos moleculares mais baixos, estreitando a curva de distribuição. Com a extensão de cadeia, ocorre aumento de peso molecular do polímero e para tempos de processamento mais

Tabela 1. Pesos moleculares e polidispersão de PEN aditivado com TPP em função do tempo de processamento

\begin{tabular}{ccccc}
\hline $\begin{array}{c}\text { TPP } \\
(\%)\end{array}$ & $\begin{array}{c}\text { Tempo de } \\
\text { processamento } \\
(\mathbf{m i n})\end{array}$ & $\begin{array}{c}\overline{\mathbf{M}} \mathbf{n} \\
(\mathrm{g} / \mathbf{m o l})\end{array}$ & $\begin{array}{c}\overline{\mathbf{M}} \mathbf{w} \\
(\mathrm{g} / \mathbf{m o l})\end{array}$ & $\overline{\mathbf{M}} \mathbf{w} / \overline{\mathbf{M}} \mathbf{n}$ \\
\hline \multirow{2}{*}{0} & 0 & 18.700 & 37.900 & 2,03 \\
& 5 & 18.200 & 33.900 & 1,86 \\
\hline & 10 & 29.400 & 51.700 & 1,76 \\
2 & 15 & 21.700 & 40.900 & 1,88 \\
& 20 & 18.200 & 35.800 & 1,96 \\
& 25 & 13.200 & 28.800 & 2,19 \\
\hline
\end{tabular}

curtos também se observa ligeira diminuição da polidispersão. À medida que o processo degradativo se inicia, a polidispersão aumenta pela quebra indefinida das moléculas de peso molecular, agora mais elevados, aumentando a polidispersão.

\section{Efeito do teor de fosfito de trifenila}

Alguns parâmetors exercem grande influência nos valores de torque observados no processamento de PEN na presença de TPP. Levando este aspecto em consideração, uma das variáveis de maior relevância é a quantidade de TPP adicionada. De acordo com a Figura 3, onde são mostradas as curvas de torque de PEN processado na presença de TPP em diferentes concentrações, foi verificado que os valores são bastante dependentes da concentração do fosfito.

Analisando as curvas obtidas para as concentrações de 1,2 e $5 \%$ de TPP, observa-se que as reações de extensão de cadeia ocorrem mais rapidamente em concentrações mais elevadas. Os valores de torque máximo são obtidos em tempos menores e a velocidade de extensão de cadeia, deduzida através da inclinação da parte ascendente da curva, é maior.

A degradação é bastante acelerada em concentrações elevadas, como a 5\% de TPP, onde já a partir de 13 ' os valores de torque são menores, quando comparados aos valores observados para o poliéster processado na ausência do fosfito. Utilizando menores concentrações de TPP, a degradação é menos acentuada e somente em tempos mais longos, os valores de torque são menores que os do polímero processado sem fosfito.

$\mathrm{O}$ aumento da velocidade de extensão de cadeia com o aumento do teor de TPP pode ser explicado pelo fato de que, com o aumento da concentração de fosfito, ocorre aumento do número de moléculas capazes de reagir com os terminais de cadeia do poliéster e promover a extensão de cadeia. Ao mesmo tempo, são

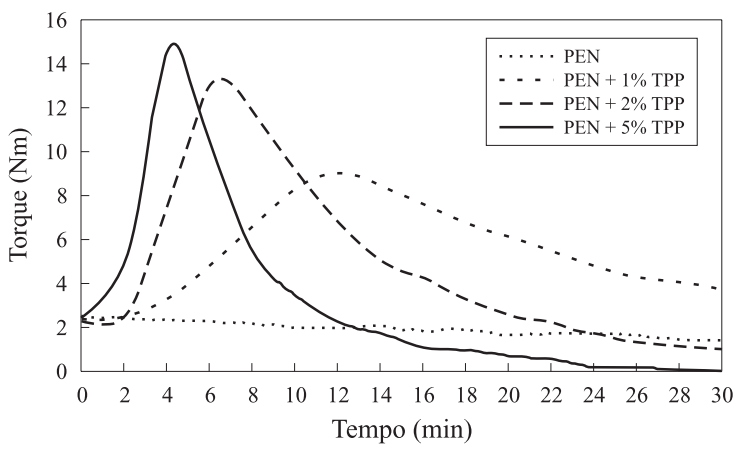

Figura 3. Efeito da concentração de TPP nas curvas de torque versus tempo de processamento de PEN a $275^{\circ} \mathrm{C}$. 
gerados maiores quantidades de subprodutos de reação, facilitando o processo degradativo posterior.

Nos estudos de Legras e colaboradore ${ }^{[12]}$ envolvendo a extensão de PET na presença de TPP, foi determinado que a adição de $1,78 \%$ de TPP equivale a uma molécula de TPP por cadeia de poliéster. Levando em consideração tal afirmação e a possível evaporação de pequena quantidade de fosfito durante a mistura, escolheu-se a concentração de $2 \%$ de TPP como a melhor concentração a ser utilizada. No caso de PEN, comparando-se os resultados obtidos neste trabalho com os de Legras, verificou-se muitas semelhanças no comportamento desses dois materiais. Entretanto, o torque máximo alcançado com adição da quantidade estequiométrica de TPP foi maior, se comparado com o uso de quantidade em excesso (5\% de TPP), resultado diferente do obtido neste trabalho. $\mathrm{O}$ peso molecular dos polímeros usados nos dois trabalhos foram próximos.

\section{Efeito da temperatura}

Outro parâmetro que influencia o processamento de PEN na presença de TPP é a temperatura. Analisando a Figura 4, onde são apresentadas corridas em três diferentes temperaturas, observa-se que em temperaturas de processamento mais baixas os valores de torque máximo são mais elevados e há menor degradação. Deve-se, entretanto, levar em consideração a diminuição da viscosidade com o aumento da temperatura. Esse efeito também contribui para a redução dos valores de torque. A diferença entre o valor final de torque das corridas é bastante significativa, enquanto que para $260^{\circ} \mathrm{C}$ altos valores de torque (aproximadamente $6 \mathrm{Nm}$ ) são mantidos até $30 \mathrm{~min}$. A $290^{\circ} \mathrm{C}$, o torque é reduzido já a partir de $10 \mathrm{~min}$ (aproximadamente $1 \mathrm{Nm}$ ). A reação é mais lenta quando ocorre em temperaturas mais baixas.

À medida que temperaturas mais elevadas são em-

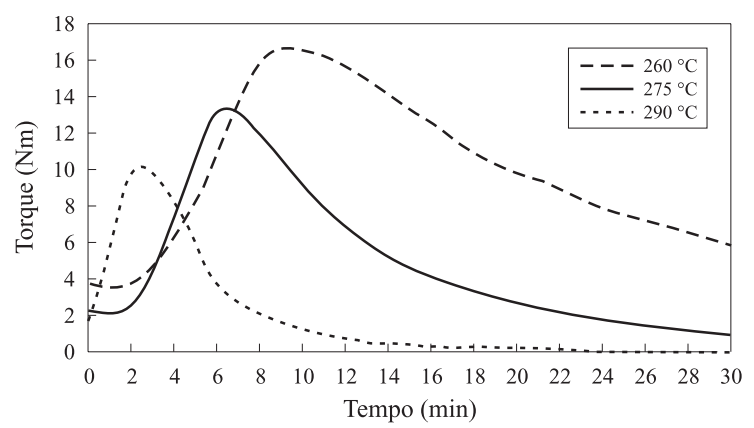

Figura 4. Efeito da temperatura nas curvas de torque versus tempo de processamento de PEN.

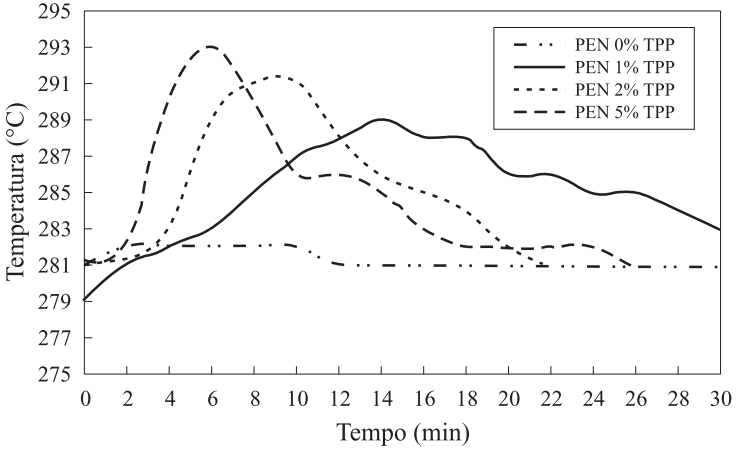

Figura 5. Temperatura da câmara de mistura versus tempo de processamento em diferentes concentrações de TPP (temperatura programada, $\left.275^{\circ} \mathrm{C}\right)$.

pregadas, a reação de extensão de cadeia ocorre mais rapidamente, porém os valores de torque máximo são mais baixos, sugerindo que as reações de degradação foram intensificadas.

A reação de extensão de cadeia estudada é um processo exotérmico, observado pelo aumento da temperatura do fundido, que eleva-se acima da temperatura de processamento programada, como apresentado na Figura 5. A concentração de TPP influencia a temperatura dentro da câmara, fazendo com que a curva de temperatura versus tempo de processamento tenha um perfil idêntico à curva de torque versus tempo de processamento.

\section{Efeito da taxa de cisalhamento}

A variação da velocidade de rotação dos rotores do misturador modifica as curvas obtidas (Figura 6). Com maiores velocidades, as quais correspondem maiores taxas de cisalhamento, as reações de extensão tendem a ser mais rápidas. $\mathrm{O}$ aumento da taxa de cisalhamento deve aumentar a posssibilidade de contato entre os sítios reativos, favorecendo a reação entre o fosfito e o polímero. Observa-se também um aumento dos processos degradativos, evidenciado pela rápida queda do torque após passar pelo ponto de máximo. Parece existir um intervalo onde a curva apresenta valores de torque mais elevados e degradação após o torque máximo não tão pronunciada. Este comportamento deve ser devido a um balanço das intensidades de extensão de cadeia e de degradação, como é visto para 60 e 80 rpm. Este aspecto não foi aprofundado, e outros estudos seriam necessários para melhor compreensão sobre o efeito do cisalhamento na curva de torque.

Outros fatores são importantes também neste processamento, como a secagem prévia do poliéster para evitar hidrólise e o tempo entre a introdução dos peletes 


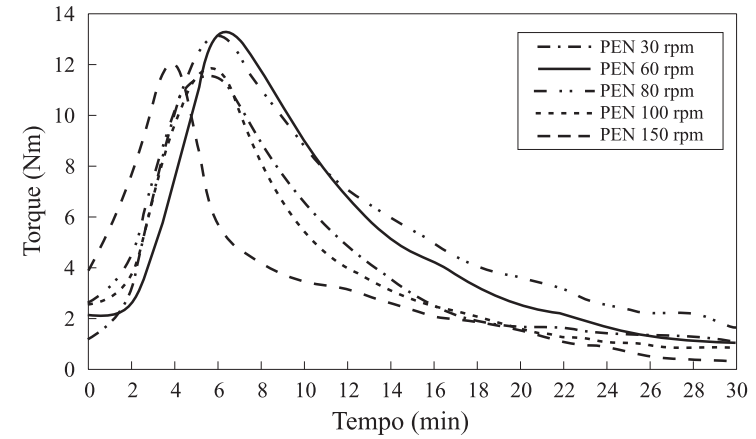

Figura 6. Efeito da velocidade dos rotores nas curvas de torque versus tempo de processamento de $\mathrm{PEN}$ a $275^{\circ} \mathrm{C}$

na câmara de mistura e a adição de TPP. Em ambos os casos, o perfil da curva é alterado devido a modificações da concentração dos terminais de cadeia do poliéster.

\section{PEN versus PET}

O processamento de PET no reômetro HAAKE nas mesmas condições anteriormente descritas revelou que o PEN apresenta valores de torque mais elevados que PET durante toda corrida de 30 minutos. Na Figura 7 são comparados os perfis da curva de torque obtida pelo processamento dos dois polímeros, com $2 \%$ de TPP e na ausência do aditivo. O PET apresenta queda mais acentuada desses valores, sugerindo uma maior suscetibilidade à processos degradativos termomecânicos catalisados pelos resíduos reacionais.

Com a adição de $2 \%$ de TPP, ocorre um aumento expressivo dos valores de torque, tanto para o PET como para o PEN. As duas curvas atingem um ponto de máximo, que no caso de PET acontece em torno de 4 minutos de processamento após a adição do fosfito e 6 minutos para o PEN, indicando que as reações de extensão de cadeia acontecem mais rapidamente para o PET. Como os polímeros apresentam o mesmo tipo de terminais de cadeia, sugere-se que a maior mobilidade

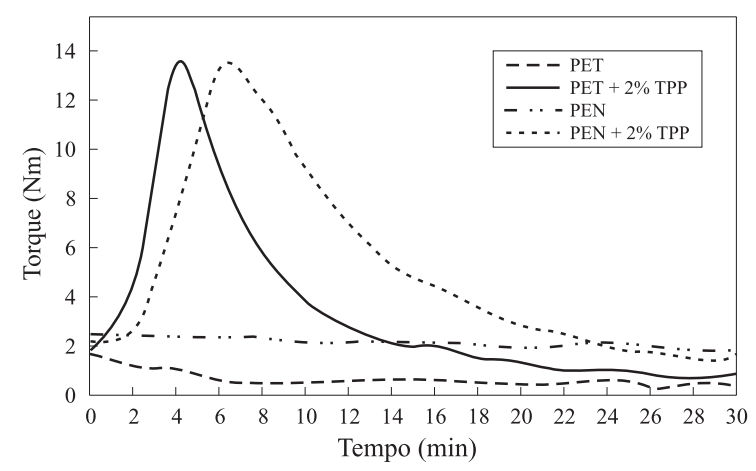

Figura 7. Curvas de torque de PET e PEN processados puros e na presença de TPP a $275^{\circ} \mathrm{C}, 60 \mathrm{rpm}$, por 30 ;. molecular do PET facilite a interação de seus terminais com o aditivo, enquanto que a estrutura rígida do PEN faz com que maiores tempos sejam necessários para as reações de extensão de cadeia. O valor de torque máximo para os dois polímeros são praticamente iguais. Após o ponto de máximo, o torque diminui continuamente com o tempo, devido a predominância de reações degradativas. O PEN é mais estável nessa etapa.

As velocidades relativas de extensão de cadeia e de degradação após o torque máximo foram estimadas a partir da inclinação das partes ascendente e descendente das curvas de torque de PET e PEN. De acordo com a inclinação das curvas, as velocidades de extensão apresentam valores de $2,71 \mathrm{Nm} / \mathrm{min}$ para o PET e $1,98 \mathrm{Nm} / \mathrm{min}$ para o PEN, confirmando que a maior velocidade de extensão de cadeia ocorre com o PET. O mesmo tipo de análise foi feita também para a parte descendente da curva, que corresponde a predominância dos processos degradativos. O PET também apresenta maior coeficiente angular $(-1,1 \mathrm{Nm} / \mathrm{min})$ que $\mathrm{o}$ PEN (-0,71 Nm/min), o que sugere ser mais suscetível a reações de quebra causada pelos subprodutos reacionais e condições de processamento.

\section{Propriedades térmicas do PEN estendido}

A influência do aditivo nas propriedades térmicas de PEN processado com TPP foi também estudada. As amostras analisadas por DSC foram obtidas após $10 \mathrm{~min}$ de processamento do PEN com diferentes concentrações de TPP. Para cada processamento, parte da amostra processada passou por processo de extração com acetona antes da análise térmica e parte foi analisada contendo o aditivo. A Tabela 2 apresenta os resultados obtidos.

Com relação as amostras de PEN que não so-

Tabela 2. Propriedades térmicas de PEN estendido com TPP, antes e após extração com acetona

\begin{tabular}{cccccc}
\hline Amostra & $\begin{array}{c}\text { TPP } \\
(\mathbf{\%})\end{array}$ & $\begin{array}{c}\mathbf{T}_{\mathbf{g}} \\
\left({ }^{\circ} \mathbf{C}\right)\end{array}$ & $\begin{array}{c}\mathbf{T}_{\mathbf{m}} \\
\left({ }^{\mathbf{C}} \mathbf{C}\right)\end{array}$ & $\begin{array}{c}\mathbf{T}_{\mathbf{c c}} \\
\left({ }^{\circ} \mathbf{C}\right)\end{array}$ & $\begin{array}{c}\mathbf{T}_{\mathbf{c h}} \\
\left({ }^{\circ} \mathbf{C}\right)\end{array}$ \\
\hline \multirow{2}{*}{ Antes da extração } & 1 & 108 & 248 & 205 & 167 \\
& 2 & 94 & 245 & 195 & 152 \\
& 5 & 76 & 235 & 190 & 131 \\
\hline \multirow{2}{*}{ Após extração } & 1 & 114 & 252 & - & 208 \\
& 2 & 111 & 253 & - & 182 \\
& 5 & 108 & 253 & 186 & 177 \\
\hline
\end{tabular}


freram extração, verificou-se que as propriedades térmicas investigadas neste estudo foram significativamente influenciadas pela presença dos resíduos do fosfito. Um efeito de plastificação, que aumenta a mobilidade segmental das cadeias, parece ter superado o efeito do aumento de peso molecular à medida que se eleva a quantidade de TPP no sistema, explicando a queda nos valores de $\mathrm{T}_{\mathrm{g}}, \mathrm{T}_{\mathrm{ch}}, \mathrm{e} \mathrm{T}_{\mathrm{cc}}$. No caso da temperatura de fusão cristalina $\left(T_{m}\right)$, a formação de estruturas cristalinas menos perfeitas, em virtude da presença de fragmentos do fosfito em regiões intraesferulíticas, é sugerida como causa do decréscimo dos valores de $\mathrm{T}_{\mathrm{m}}$ com o aumento da concentração de TPP em amostras não-extraídas.

Nas amostras de PEN que sofreram extração, onde os subprodutos da reação com TPP foram removidos, as diferenças são menos marcantes. $\mathrm{A}_{\mathrm{g}}$ e a $\mathrm{T}_{\mathrm{ch}}$ sofreram pequena redução. A diminuição da $T_{g}$, observada principalmente em amostras processadas com maiores teores de TPP, pode ser devida a ineficiente remoção dos resíduos do aditivo pela extração com acetona. Assim, algum efeito de plastificação ainda estaria ocorrendo. A presença dessa pequena quantidade de resíduo, que pode atuar como agente nucleante, pode ter sido responsável pela pequena diminuição observada para a $T_{c h}$, que é indicativo de um aumento na velocidade de cristalização. A $T_{m}$ praticamente não se altera. A $\mathrm{T}_{\mathrm{cc}}$ só aparece para concentrações elevadas de TPP (5\%), o que de certa forma confirma que algum resíduo permaneceu na amostra após a extração. Esses resíduos devem ter promovido a cristalização da amostra de mais alto peso molecular, que não foi possível quando menores concentrações de TPP foram empregadas para a reação de extensão de cadeia.

\section{Conclusões}

Na presença de TPP, o processamento de PEN resulta em aumento da massa molecular do polímero em virtude da ocorrência de reações de extensão de cadeia catalisadas pelo fosfito. A massa molecular do poliéster aumenta com o aumento do tempo de processamento e apresenta um máximo, após o qual reações de degradação promovidas pela presença de subprodutos da extensão de cadeia passam a predominar. A intensidade das reações de extensão de cadeia aumenta com o aumento do teor de TPP, da temperatura e do cisalhamento. A velocidade das reações de extensão e degradação é maior para PET do que para o PEN.

\section{Agradecimentos}

Os autores agradecem ao PADCT/FINEP, ao CNPq e a Rhodia-Ster e Hoescht pela doação do PEN e do PET utilizados neste estudo.

\section{Referências Bibliográficas}

1. Myers, J. - Modern Plastic International, 23(12), p.30 (1993).

2. Ophir, A.; Kenig, S.; Shai, A.; Barka'ai, Y. - ANTEC'99, p.1010 (1999).

3. Harazoe, H.; Sakai, M. - Patente Americana 5.573.820 (1996).

4. Sohumann, H.D.; Thiele, U. - Patente Americana 5.656.221 (1997).

5. Stouff, J.M., Blanchard, E.N.; Leffew, K.W. - Patente Americana 5.540.868 (1996).

6. Inata, H.; Matsumura, S. - J. Appl. Polym. Sci., 30, p.3325 (1985).

7. Aharoni, S.M.; Forbes, C.E.; Hammond, W.B.; Hindenlang, D.M.; Markes, F.; O’Brien, K. - J. Polym. Sci., Polym. Chem., 24, p.1281 (1986).

8. Devaux, J.; Godard, P.; Mercier, J.P. - J. Polym. Sci., Polym. Phys., 20, p.1901 (1982).

9. Jacques, B.; Devaux, J.; Legras, R. - Polymer, 37(7), p.1189 (1996).

10. Jacques, B.; Devaux, J.; Legras, R. - Macromolecules, 29, p.3129 (1996).

11. Jacques, B.; Devaux, J.; Legras, R. - Makromol. Chem.Symp., 75, p.231 (1993).

12. Jacques, B.; Devaux, J.; Legras, R. - Polymer, 37 (18) p.4085 (1996).

13. Nascimento, C.R.; Dias, M.L. - J. Polym. Eng., 20(2), p.143 (2000).

14. Silva, A.P.F.; Dias, M.L. - Polym. Eng. Sci., 40(8), p.1777 (2000).

15. Nascimento, C.R. - "Reciclagem de garrafas de PET na presença de fosfitos orgânicos”, Tese de Mestrado, Universidade Federal do Rio de Janeiro, Brasil, 1997.

16. Silva, S.M.L. - "Processamento reativo da misturas de PET/poliamida-6 na presença de fosfito orgânico”, Tese de Doutorado, Universidade Federal do Rio de Janeiro, Brasil, 2000.

17. Aharoni, S.M. - Int. J. Polym. Mat., 26, p.9 (1994).

Recebido: 04/01/01

Aprovado:10/09/01 